\title{
Tailoring an Evidence-Based Practice Fellowship to Meet Learning Needs of Bedside Nurses
}

Donna J. Plonczynski ${ }^{1^{*}}$ and Donna Kruse ${ }^{2}$ RN, MS, CCRN-CMC-CSC

${ }^{1}$ Associate professor, Northern Illinois University, USA

${ }^{2}$ Clinical Educator/Magnet Program Director, Advocate Sherman Hospital, USA

"Corresponding author: Donna J. Plonczynski, Associate professor, Northern Illinois University, 1240 Normal Rd, DeKalb, IL 60115, USA, Tel: 815.753.6584; Fax: 815.753.0814; E-mail: djplonz@niu.edu

Received date: August 11, 2014, Accepted date: September 19, 2014, Publication date: November 27, 2014

Copyright: (c) 2014 Plonczynski DJ, et al. This is an open-access article distributed under the terms of the Creative Commons Attribution License, which permits unrestricted use, distribution, and reproduction in any medium, provided the original author and source are credited.

\begin{abstract}
The ability for nurses to practice to the fullest level of their education and expertise in order to optimize patient care is an ideal vision for nursing practice. While healthcare quality has improved over the past decades, delays continue in transferring current information from research to patient care. Many nurses are not experienced in transferring research into practice. This paper describes the process for planning, implementing and evaluating the evidence-based practice (EBP) Fellowship that transferred these critical skills to five bedside nurses. All five Fellows implemented evidence in their area of practice with significant positive changes in patient outcomes. The results from the Fellowship indicate that we met and exceeded the goals of transferring the knowledge, skills and attitudes for EBP to bedside nurses. Unexpected outcomes included the development of leadership and presentation skills as all five of the Fellows disseminated the results of their successful projects regionally and nationally.
\end{abstract}

\section{Background}

In a powerful Lancet article, it was estimated that overall $20-25 \%$ of patients receive treatments are that are either unnecessary or potentially harmful and that $30-40 \%$ patients do not receive evidencebased practice (EBP) care [1]. The United States Department of Health and Human Services (DHHS) has prioritized "high value, safe and effective health care" for U.S. healthcare systems [2]. The Department has set goals to be met by the end of 2015 toward the decline of preventable injuries in hospitals by $40 \%$, as well as the reduction of readmissions by $20 \%$ annually. The realization of these goals could save the lives of 60,000 patients as well as prevent over 1.5 million patients from complications annually. This goal would also reduce Medicare costs by $\$ 50$ billion dollars over 10 years, in addition to the reduction in other payer's costs.

\section{Literature Review}

It is well established that patients are admitted to acute care hospitals because they are in need of nursing care to address their health status or manage an acute health problem. The quality of that nursing care is based on the nurse's education, as well as the state of the environment in which she works [3]. According to the National Sample Survey of Registered Nurses, the average age of registered nurses in the United States is 46 years of age [4]. Most of these nurses have graduated decades earlier in programs that did not have an emphasis on research application or the implementation of EBP. Because of this gap in professional education, many nurses are not adept at interpreting current healthcare research or using the results to change practice in a timely manner.

The environment in which the nurse works is also being evaluated in order to address discrepancies. The Agency for Healthcare Quality and Research Quality publishes a report that compares quality of hospital outcomes over a decade [5]. This recent report has demonstrated improvements, but important disparities remain. In this analysis, no hospitals worsened, and a few showed no improvement at all over the previous decade. However, $77 \%$ of hospitals that have demonstrated overall improvement over the past 10 years continue to have safety concerns. A mere $38 \%$ of hospitals demonstrated improvement on their safety measures.

The Institute of Medicine (IOM) articulated a vision for nursing care that fosters practice to the fullest level in order to optimize care [6]. While healthcare quality has improved over the past decade, delays in transferring research to the bedside continue. A recent survey indicated that nurses are interested in - and are hopeful of - being active in EBP [7]. Because nurses are interested in providing optimal care, resources are needed in order to address the skills and knowledge needed to positively impact care throughout a hospital and healthcare system. Robert Wood Johnson advocates for partnerships to assist with the advancing of knowledge and education for nurses [8].

The implementation of an EBP nursing Fellowship is an ideal mechanism to deliver evolving healthcare knowledge into practice for the benefit of hospitalized patients. While evidence-based nursing practice is a priority for nursing care, the development of Fellowship mentoring programs are evolving. One Midwestern community hospital conducts a one-year researchship to educate 8 Fellows annually on the generation of research by conducting studies within the hospital [9]. Other hospitals have changed from this type of Fellowship into an EBP model that translates current research into nursing practice $[10,11]$. One system shortened the timeline of the Fellowship to less than one year in order to implement changes more quickly [12]. Because of the time-intensive nature of the education and mentoring, along with a safety imperative, Fellowships include limited numbers of Fellows. This paper will describe the process by which a tailored Fellowship was developed, maintained, and evaluated in order to address bedside nurse's educational and environmental needs. 


\section{Methods}

This Fellowship plan began with a vision by nursing administrative leadership. The question posed to the Council of Application of Research and Evidence (CARE) was: "What are the most effective methods to conduct an ideal EBP program in order to address the learning needs of bedside nurses in order to improve patient outcomes?" The draft outline of the Fellowship was determined from published literature and from practices at other hospitals and academic centers [11]. It was tailored to meet the needs of this hospital's nurses. Brainstorming, a draft proposal, and subsequent revisions as a Council were developed as a result of this challenge from nursing leadership.

The purpose of the nursing Fellowship program was to promote quality care for the patients by providing the knowledge, skills and attitudes to bedside nurses for implementation of EBP. This purpose was accomplished by the incorporation of research into practice, which will close the time delay now seen nationwide between knowledge generation and its implementation.

The reality of healthcare practice includes the need for funding. Funding was needed for a research and statistical consultant, the salary of 92 work hours per Fellow, supplies, and the costs of poster generation and conference attendance for subsequent presentations. Disseminating the results of the EBP was critical to the program's success. The hospital sought and obtained funding from a local community foundation and a local benefactor. Similar support from the hospital included technological, logistic, and coordinator support for the duration of the program. In addition, continuing education credits that count toward re-licensure were provided for the Fellows through the hospital.

A foundational consideration was the choice of a model used for the program. The process by which research is initially identified, transferred throughout the nursing staff, and used in patient care follows a nursing practice framework. Multiple clinical and academic settings use the Iowa Model $[13,14]$, which uses an algorhythm that nurses "find intuitively understandable" [15]. The well-established Iowa model and educational program were used as a template for the Fellowship after evaluating multiple other models [14].

The modifications of the original educational program from a more research-focus are listed below [16]. These modifications were made in keeping with cost accountability and timeline considerations:

- timeline reduced from one-to-two years to three months;

- reduced class meetings from 12 to 4 days;

- placed locating evidence on first day, rather than third;

- removed conference attendance as part of initial Fellowship timeline;

- added discussions each meeting to foster collaboration and problem-solving as a group; and

- included creation and presentation of poster and oral presentation to leaders and mentors as part of the Fellowship timeline.

Once the plan was complete and funding obtained, the application and review process were developed by the CARE council. The application form included suggested topics for an EBP project, but choosing one of the suggested topics was not used as a criterion for selection. The application process was announced via the hospital's newsletter, emails and nursing forums and was available on-line and in paper formats in various locations. Each applicant submitted an interest statement, an outline of previous professional changes he/she had implemented and their outcomes, as well as an EBP project idea. Completed applications were blinded and forward to the CARE council members by the chair for review and scoring. Applicants were then notified via letter of results and comments. Those applicants that were not selected were given feedback on methods to improve their application for the next submission.

Each chosen Fellow obtained a letter of support from his/her leader and signed a contract to ensure clear communication of expectations. Each Fellow agreed to attend and participate at all EBP workshop sessions. In addition, they agreed to meet individually with their identified mentor and nurse leader at least one hour per month. Furthermore, they agreed to complete the EBP project in the three months of the Fellowship and promote the further implementation of their EBP across departments and committees in subsequent months. Finally, the Fellows agreed to develop and deliver a poster and podium presentation and submit their work for a regional or national conference.

The five selected Fellows were mentored for 92 hours over three months. The Fellow was provided with 32 hours of workshop time and used the remaining 60 hours toward mentored implementation and evaluation of the new practice. The Fellowship hours were separate from his/her usual practice responsibilities on the department schedule and did not count toward the departmental productivity goals.

It was determined that the Fellowship was best presented in a workshop format with an application focus with each topic. The consideration of the program as a workshop was critical to its success for bedside nurses. Each presentation, whether on searching literature, evaluating literature, or entering excel data was followed by a mentored practice session on computers with Internet access.

Our topics were modified for bedside nurses. The following were topics covered during the workshops:

\begin{tabular}{|l|}
\hline Organizational and leadership support of EBP \\
\hline The lowa Model \\
\hline $\begin{array}{l}\text { Defining the EBP project's development with PICOT (population, intervention, } \\
\text { control or comparison group, outcome, and timing) }\end{array}$ \\
\hline Action plan \\
\hline Feasibility analysis and team building \\
\hline Literature and web resource searching \\
\hline Evaluating resources for quality \\
\hline Obtain evidence (baseline data) \\
\hline Excel data entry \\
\hline Role of statistics \\
\hline Writing a protocol/procedure/guideline \\
\hline Implementing change \\
\hline Poster and podium presentation development \\
\hline
\end{tabular}

Table 1: Topics covered during the workshops. 
Application to practice was the rationale for reducing sections on literature reviews and literature synthesis. For example, there was no use of research critique forms. This Fellowship did not include some models such as the research translation model, nor did it include research language such as independent and dependent variables. The focus of the research parts of this Fellowship focused on the use of high-quality evidence, such as from internationally recognized sources. The purpose of the statistical presentation was to enhance understanding of statistics without explaining how to conduct the analyses.

Our modifications also included the use of notebooks to track progress, ideas, and barriers for problem solving by the individual with the mentor or with the Fellowship group. Each workshop concluded with a review of assignments that were to be completed prior to the next meeting.

To ensure that the process was understandable, the planner and major presenter continually linked the EBP process to the nursing process of assesses, diagnose, plan, intervene and evaluate, which was labeled the 'Iowa Model in a nutshell' : (Table 2)

\begin{tabular}{|l|l|}
\hline Steps in EBP process & Nursing process \\
\hline $\begin{array}{l}\text { Identify an issue of concern; and } \\
\text { Assemble and evaluate evidence in literature and } \\
\text { current best practices }\end{array}$ & Assess \\
\hline $\begin{array}{l}\text { Measure baseline data and determine exact issue } \\
\text { that can be addressed }\end{array}$ & Diagnose \\
\hline $\begin{array}{l}\text { Develop implementation and outcome measurement } \\
\text { steps }\end{array}$ & Plan \\
\hline Pilot protocol & Implement \\
\hline Measure process and outcomes & Evaluate \\
\hline
\end{tabular}

Table 2: Iowa Model in a nutshell.

The goals of this Fellowship were accomplished in three months with meetings held approximately every three weeks. The remaining time was spent on individual mentored development of the project. A recognition event was held at the end of the three months with mentors and leaders. At that time, Fellows presented their posters and a podium presentation to the leadership, followed by several weeks of presentations to various audiences, including the funders, the hospital staff and medical staff.

\section{Results}

The projects all were a success in many ways. There were significant improvements in each of the five EBP changes. The projects:

- reduced delirium by using a wake-up protocol for patients who were intubated;

- improved breastfeeding rates and maternal bonding by allowing for daily maternal nap and quiet time;

- provided pain relief for children using distraction techniques on Ipads@;

- improved discharges to home instead of to extended care facilities by developing a comprehensive walking program; and

- reduced the risk of errors by implementing a medication-passing program.
The feedback from the Fellows at each workshop was positive. The total of the feedback forms $(n=75)$ from the Fellows on the evaluation of each of the seven presenter's effectiveness was labeled to a 'great extent' $(95 \%, \mathrm{n}=71)$, 'moderate extent' $(4 \%, \mathrm{n}=3)$, and 'slight extent' $(1 \%, n=1)$. For the summed feedback $(n=30)$ on the extent course objectives were met each workshop, $90 \%(n=27)$ stated 'great extent', while $10 \%(\mathrm{n}=3)$ stated 'moderate extent'.

At the conclusion of the Fellowship, the Fellows were asked to provide overall evaluations, as well as barriers and facilitators to the program. The comments were as follows: 'Excellent'; 'Met and exceeded goals'; 'Improved confidence as nurse'; 'Improved ability to publically speak'; 'Added leadership role to department'; 'Improved written communication skills'; 'Expanded understanding of research'.

The two barriers identified by the Fellows were difficulties scheduling off department time and the short time to complete the project. The three identified facilitators were 'Excellent feedback'; 'Support systems: Mentors, Leaders, Coordinator'; and 'Resources for getting project done'.

To determine the impact of the Fellowship further out in time, the Fellows, Mentors and Leaders were asked to anonymously critique the program six months after its completion. By that time, all the projects had been expanded or were in the process of being expanded in other departments. Additionally, several Fellows had joined the CARE council and had been active in promoting EBP in units. All of the Fellows had presented their posters and PowerPoint's locally, and all were accepted to present at a national EBP conference. Furthermore, two of the Fellows had enrolled in Advanced Practice Nurse (APN) Master's degree programs.

One leader wrote in 6-month follow-up evaluation that this was a "Great Fellowship, I'm glad to see it's continued. I believe this will shape nursing practice at this Hospital for the better in the future, and will plant the seeds for many great changes." Furthermore, the Chief Nursing Officer (CNO), who had originated this program's vision provided feedback. She stated that the Fellowship "Had a positive impact on patient outcomes; increased the value of EBP in our organization; provided new opportunities for poster and project presentations in the region and nationally; and elevated/advanced the practice of nursing".

\section{Discussion}

This Fellowship met the DHHS goals of providing safe, effective patient care that is cost-effective [2]. In hospitals, because the care provided by nurses is instrumental for optimal patient outcomes, the use of EBP by nurses is crucial to meeting the DHHS goals. In each project, the Fellow demonstrated improved patient outcomes with EBP care.

This Fellowship was in keeping with Robert Wood Johnson's [8] encouragement of interdisciplinary partnerships to advance knowledge for nurses, with the use of an informaticist, educator, researcher, case manager, data manager, and coordinator.

These Fellows volunteered for the program and demonstrated great interest in being active in EBP, similar to what is found in the literature [7]. Because nurses are interested in providing optimal care, resources are needed in order to address the skills and knowledge needed to positively impact care throughout a hospital and healthcare system. Financing and health system resources were made available 
through administrative and community support. This was a critical factor in the program's success.

The Fellowship fostered improvements in patient care at multiple levels by implementing EBP rapidly to the bedside. This result is in keeping with the IOM's vision for nursing to provide practice at an optimal level to ensure safe and effective patient outcomes [6].

In answering the CNO's challenge about her vision for evidencebased nursing practice, the CARE council implemented the Fellowship outline from published literature and from best practices at other hospitals and academic centers [11]. Because most of the Fellows did not have experience in EBP implementation, the Fellowship required a tailored, bedside-focused workshop format.

The results from the Fellowships indicate that the goals of providing the knowledge, skills and attitudes to bedside nurses for implementation of EBP were met and exceeded. The mentored process has further developed these excellent nurses and recognized them for their investment in optimizing patient care. Additionally, the work of the Fellows was at such a high level, that they each have presented their work regionally and nationally. A final, and unexpected result of the Fellowship was the development of leadership skills in these nurses, who have gone on to mentor other nurses, pursue further degrees, and assumed membership in health system committees.

\section{Future implications}

The positive transformational impact of mentoring process on nurses has been well recorded in residency programs [17], but not in EBP Fellowships. Therefore, an analysis of the impact on process and outcome measures such as leadership roles and nurse retention has yet to be studied extensively. Research on these issues would help direct future programs.

For this Fellowship, the facilitators and barriers as well as feedback from the Fellows, Mentors, and Leaders were used to further develop the structure of the Fellowship. With Fellows now serving on the CARE council, their feedback will be used in future planning, thereby closing the feedback loop. This process further supports the culture of EBP in the health system.

\section{Acknowledgements}

The Fellows who have successfully navigated EBP changes.

Their leaders and mentors

Judy Balcitis, RN, MSN, CNO

Benefactors for the program funding

\section{References}

1. Grol R, Grimshaw J (2003) From best evidence to best practice: effective implementation of change in patients' care. Lancet 362: 1225-1230.

2. U.S. Department of Health and Human Services (DHHS, 2010). Strategic plan and priorities: Fiscal years 2010-2015.

3. Aiken, L., Cimiotti, J., Sloan, D., Smith, H., Flynn, L., \& Neff, D (2011) Effects of nurse staffing and nurse education on patient deaths in hospitals with different nurse work environments. Medical Care, 49(12): 1047-1053.

4. Federal Division of Nursing (2010) The registered nurse population: Findings from the 2008 national sample survey of Registered Nurses. Department of Health and Human Services, Washington, USA.

5. Rockville MD (2012) Agency for Healthcare Research and Quality. National healthcare quality report.

6. Institute of Medicine (2010) The Future of nursing: Leading change, advancing health. National Academies Press Washington, USA.

7. Soukup M, McCleish J (2008) Advancing evidence-based practice: a program series. J Contin Educ Nurs 39: 402-406.

8. Robert Wood Johnson (2011) The nursing profession: development, challenges, and opportunities. Mason D, Isaacs S and Colby D (Eds.), Wiley, Jossey-Bass: Hoboken, USA.

9. Turkel MC, Ferket K, Reidinger G, Beatty DE (2008) Building a nursing research fellowship in a community hospital. Nurs Econ 26: 26-34.

10. Gattusso, J., Hinds, P., Beaumont, C., Funk, A., Green, J., et al. (2007) Transforming a hospital nursing research fellowship into an evidencebased practice fellowship. Journal of Nursing Administration, 37 : 539-545.

11. Weeks S, Moore P, Allender M (2011) A regional evidence-based practice Fellowship: Collaborating competitors. Journal of Nursing Administration, 41: 10-14.

12. Milne D, Krishnasamy M, Aranda S (2007) Promoting evidence-based care through a clinical research fellowship programe. Journal of Clinical Nursing 16: 1629-1639.

13. Krom Z, Batten J, Bautista C (2010) A Unique Collaborative Nursing Evidence-Based Practice Initiative Using the Iowa Model: A Clinical Nurse Specialist, a Health Science Librarian, and a Staff Nurse's Success Story. Clinical Nurse Specialist 24: 54-59.

14. Titler MG, Kleiber C, Steelman VJ, Rakel BA, Budreau G, et al. (2001) The Iowa Model of Evidence-Based Practice to Promote Quality Care. Crit Care Nurs Clin North Am 13: 497-509.

15. Gawlinski A, Rutledge D (2008) Selecting a model for evidence-based practice changes: a practical approach. AACN Adv Crit Care 19: 291-300.

16. Cullen L, Titler MG (2004) Promoting evidence-based practice: an internship for staff nurses. Worldviews Evid Based Nurs 1: 215-223.

17. Kramer M, Maguire P, Halfer D, Budin WC, Hall DS, et al. (2012) The organizational transformative power of nurse residency programs. Nurs Adm Q 36: 155-168. 